Supplement of The Cryosphere, 10, 1433-1448, 2016

http://www.the-cryosphere.net/10/1433/2016/

doi:10.5194/tc-10-1433-2016-supplement

(C) Author(s) 2016. CC Attribution 3.0 License.

(c) (i)

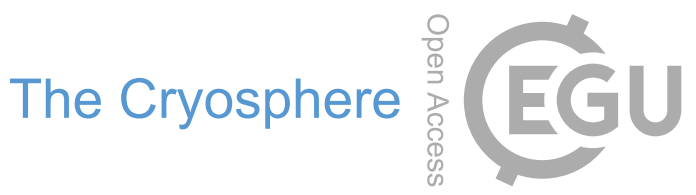

Supplement of

\title{
Glacier melting and precipitation trends detected by surface area changes in Himalayan ponds
}

\section{F. Salerno et al.}

Correspondence to: Franco Salerno (salerno@irsa.cnr.it)

The copyright of individual parts of the supplement might differ from the CC-BY 3.0 licence. 


\section{Supporting Information}

Table SII List of gridded and reanalysis data investigated in this study with relevant technical specifications.

\begin{tabular}{|c|c|c|c|c|c|c|c|c|c|}
\hline $\begin{array}{l}\text { Meteorological } \\
\text { variables }\end{array}$ & Product & Version & $\begin{array}{l}\text { Temporal } \\
\text { resolution }\end{array}$ & $\begin{array}{l}\text { Temporal } \\
\text { coverage }\end{array}$ & $\begin{array}{l}\text { Spatial } \\
\text { resolution }\end{array}$ & $\begin{array}{c}\text { Spatial } \\
\text { coverage }\end{array}$ & Produced by & Web site & Reference \\
\hline \multicolumn{10}{|l|}{ Gridded data } \\
\hline Temperature & $\begin{array}{l}\text { GHCN CAMS } \\
\text { (Global Historical } \\
\text { Climatology Centre, } \\
\text { the Climate Anomaly } \\
\text { Monitoring System) }\end{array}$ & - & monthly & 1948-present & $0.5^{\circ}$ & $\begin{array}{l}\text { Global land } \\
\left(89.75^{\circ} \mathrm{S}-\right. \\
89.75^{\circ} \mathrm{N} \text {, } \\
0.25^{\circ} \mathrm{E}- \\
\left.359.75^{\circ} \mathrm{E}\right)\end{array}$ & $\begin{array}{l}\text { Climate Prediction Center (CPC) } \\
\text { of the National Centers for } \\
\text { Environmental Prediction } \\
\text { (NCEP). }\end{array}$ & $\begin{array}{l}\text { htttp://www.esrl.noaa.gov/psd/data/gridded/ } \\
\text { data.ghcncams.httml }\end{array}$ & $\begin{array}{l}\text { Fan, van den } \\
\text { Dool (2008) }\end{array}$ \\
\hline $\begin{array}{l}\text { Temperature/Precipitati } \\
\text { on }\end{array}$ & $\begin{array}{l}\text { CRU TS (Climate } \\
\text { Research Unit -Time } \\
\text { Series) }\end{array}$ & V3.2 & monthly & 1901-2011 & $0.5^{\circ}$ & Global & $\begin{array}{l}\text { Climate Research Unit - } \\
\text { University of East Anglia }\end{array}$ & $\begin{array}{l}\text { http://badc.nerc.ac.uk/view/badc.nerc.ac.u } \\
\text { k_ATOM_ACTIVITY_3ec0d1c6-4616- } \\
\text { 11e2-89a3-00163e251233 }\end{array}$ & $\begin{array}{l}\text { Harris et al. } \\
(2015)\end{array}$ \\
\hline Precipitation & APHRODITE & V1101 & daily & $1951-2007$ & $0.25^{\circ}$ & $\begin{array}{l}\text { Regional land } \\
\left(15^{\circ} \mathrm{S}-55^{\circ} \mathrm{N},\right. \\
\left.60^{\circ} \mathrm{E}-150^{\circ} \mathrm{E}\right)\end{array}$ & $\begin{array}{l}\text { APHRODITE (Asian } \\
\text { Precipitation Highly Resolved } \\
\text { Observational Data Integration } \\
\text { Towards Evaluation of Water } \\
\text { Resources) project in } \\
\text { collaboration with the Research } \\
\text { Institute for Humanity and Nature } \\
\text { and the Meteorological Research } \\
\text { Institute of the Japan } \\
\text { Meteorological Agency }\end{array}$ & $\begin{array}{l}\text { http://www.chikyu.ac.jp/precip/products/in } \\
\text { dex.html }\end{array}$ & $\begin{array}{l}\text { Yatagai et al. } \\
\text { (2012) }\end{array}$ \\
\hline Precipitation & $\begin{array}{l}\text { GPCC (Global } \\
\text { Precipitation } \\
\text { Climatology Centre) }\end{array}$ & V6 & monthly & $1901-2010$ & $0.5^{\circ}$ & Global & $\begin{array}{l}\text { Deutscher Wetterdienst (National } \\
\text { Meteorological Service of } \\
\text { Germany) in the framework of the } \\
\text { World Climate Research Program }\end{array}$ & http://www.esrl.noaa.gov/psd/ & $\begin{array}{l}\text { Schneider et al. } \\
\text { (2013) }\end{array}$ \\
\hline \multicolumn{10}{|l|}{ Reanalysis data } \\
\hline $\begin{array}{l}\text { Temperature/Precipitati } \\
\text { on }\end{array}$ & $\begin{array}{l}\text { NCEP CFS (National } \\
\text { Centers for } \\
\text { Environmental } \\
\text { Prediction- Climate } \\
\text { Forecast System) }\end{array}$ & V2 & hourly & 1979-present & $0.5^{\circ}$ & Global & $\begin{array}{l}\text { National Centers for } \\
\text { Environmental Prediction } \\
\text { (NCEP) }\end{array}$ & http://cfs.ncep.noaa.gov/cfsr/ & $\begin{array}{l}\text { Saha et al. } \\
\text { (2010) }\end{array}$ \\
\hline $\begin{array}{l}\text { Temperature/Precipitati } \\
\text { on }\end{array}$ & ERA Interim & - & 6-hourly & 1979-present & $0.75^{\circ}$ & Global & $\begin{array}{l}\text { ECMWF (European Centre for } \\
\text { Medium-Range Weather } \\
\text { Forecasts) }\end{array}$ & $\begin{array}{l}\text { http://apps.ecmwf.int/datasets/data/interim- } \\
\text { full-daily/levtype=sfc/ }\end{array}$ & - Dee et al. (2011) \\
\hline
\end{tabular}


Table SI2. Data sources used for tracing the inter-annual variations of glaciers and ponds since the early 1960s

\begin{tabular}{|c|c|c|c|c|c|}
\hline $\begin{array}{l}\text { Abbreviation } \\
\text { used in the text }\end{array}$ & $\begin{array}{l}\text { Topographic } \\
\text { map }\end{array}$ & $\begin{array}{l}\text { Acquisition } \\
\text { date }\end{array}$ & Scale & \multicolumn{2}{|c|}{ Acquisition technique } \\
\hline TISmap-63 & $\begin{array}{l}\text { Topographic map } \\
\text { of Indian Survey }\end{array}$ & 1963 & $1: 50000$ & \multicolumn{2}{|c|}{$\begin{array}{l}\text { Vertical aerial photographic survey 1957-1959 } \\
\text { and field survey in } 1963 \text { (Yamada, 1998) }\end{array}$} \\
\hline $\begin{array}{l}\text { Abbreviation } \\
\text { used in the text }\end{array}$ & $\begin{array}{l}\text { Satellite } \\
\text { image }\end{array}$ & $\begin{array}{l}\text { Acquisition } \\
\text { date }\end{array}$ & $\begin{array}{l}\text { Spatial } \\
\text { resolution }(\mathrm{m})\end{array}$ & Sensor & Scene ID \\
\hline Landsat-92 & Landsat 5 & 17 Nov 1992 & 30 & TM & ETP140R41_5T19921117 \\
\hline Landsat-00 & Landsat 7 & 30 Oct 2000 & $15^{\mathrm{a}}$ & ETM+ & LE71400412000304SGS00 \\
\hline ALOS-08 & ALOS & 24 Oct 2008 & 10 & AVNIR-2 & ALAV2A146473040 \\
\hline Landsat-11 & Landsat 7 & 30 Nov 2011 & $15^{\mathrm{a}, \mathrm{b}}$ & ETM+ & LE71400412011334EDC00 \\
\hline Landsat-13 & Landsat 8 & 10 Oct 2013 & $15^{\mathrm{a}}$ & OLI & LC81400412013283LGN00 \\
\hline
\end{tabular}

Table SI3. Data sources used for the inter-annual variations analysis carried out during the 2000-2013 period

\begin{tabular}{|c|c|c|c|c|}
\hline $\begin{array}{l}\text { Satellite } \\
\text { image }\end{array}$ & Acquisition date & Spatial resolution (m) & Sensor & Scene ID \\
\hline \multirow[t]{13}{*}{ Landsat 7} & 30 Oct 2000 & 15 (pan sharpened) & ETM+ & LE71400412000304SGS00 \\
\hline & 17 Oct 2001 & & & LE71400412001290SGS00 \\
\hline & 20 Oct 2002 & & & LE71400412002293SGS00 \\
\hline & 8 Nov 2003 & & & LE71400412003312ASN01 \\
\hline & 10 Nov 2004 & & & LE71400412004315PFS00 \\
\hline & 28 Oct 2005 & & & LE71400412005301PFS00 \\
\hline & 16 Nov 2006 & & & LE71400412006320PFS00 \\
\hline & 19 Nov 2007 & & & LE71400412007323PFS00 \\
\hline & 07 Dec 2008 & & & LE71400412008342SGS00 \\
\hline & 08 Nov 2009 & & & LE71400412009312SGS00 \\
\hline & 11 Nov 2010 & & & LE71400412010315PFS00 \\
\hline & 30 Nov 2011 & & & LE71400412011334EDC00 \\
\hline & 17 Nov 2012 & & & LE71400412012321PFS00 \\
\hline Landsat 8 & 10 Oct 2013 & 15 (pan sharpened) & OLI & LC81400412013283LGN00 \\
\hline
\end{tabular}

Table SI4. Data sources used for the intra-annual variations analysis carried out during the 2001 year

\begin{tabular}{|c|c|c|c|c|}
\hline $\begin{array}{l}\text { Satellite } \\
\text { image }\end{array}$ & Spatial Resolution (m) & Sensor & Acquisition date & Scene ID \\
\hline \multirow[t]{5}{*}{ Landsat 7} & 15 (pan sharpened) & ETM+ & 11 Jun 2001 & LE71400412001162SGS00 \\
\hline & & & 14 Aug 2001 & LE71400412001226SGS00 \\
\hline & & & 15 Sep 2001 & LE71400412001258SGS00 \\
\hline & & & 17 Oct 2001 & LE71400412001290SGS00 \\
\hline & & & 20 Dec 2001 & LE71400412001354SGS00 \\
\hline
\end{tabular}


Table SI5. Correlation Coefficient Matrix between pond surface area variations during the 2000-2013 period and selected drivers of change.

\begin{tabular}{|c|c|c|c|c|c|c|c|c|c|c|c|}
\hline Driver/Pond Code & & LCN139 & LCN93 & LCN141 & LCN11 & LCN77 & LCN76 & LCN24 & LCN9 & LCN3 & LCN68 \\
\hline \multirow{4}{*}{$\begin{array}{c}\text { Mean Temperature } \\
\text { (Tmean) }\end{array}$} & pre- & -0.40 & -0.53 & -0.51 & -0.34 & -0.45 & -0.51 & -0.06 & -0.04 & -0.10 & -0.18 \\
\hline & monsoon & 0.02 & 0.18 & 0.14 & 0.27 & 0.05 & 0.15 & 0.54 & 0.08 & 0.32 & 0.09 \\
\hline & post- & -0.41 & -0.36 & -0.27 & -0.35 & -0.20 & -0.29 & 0.06 & -0.03 & -0.04 & -0.24 \\
\hline & annual & -0.47 & -0.52 & -0.45 & -0.37 & -0.36 & -0.42 & 0.08 & -0.06 & -0.10 & -0.29 \\
\hline \multirow{4}{*}{$\begin{array}{l}\text { Minimum } \\
\text { Temperature }\end{array}$} & pre- & -0.51 & -0.52 & -0.54 & -0.43 & -0.52 & -0.54 & -0.15 & -0.26 & -0.33 & -0.40 \\
\hline & monsoon & -0.31 & -0.20 & -0.25 & -0.17 & -0.23 & -0.05 & 0.21 & -0.40 & -0.38 & -0.42 \\
\hline & post- & -0.49 & -0.47 & -0.45 & -0.51 & -0.32 & -0.37 & -0.02 & -0.18 & -0.27 & -0.36 \\
\hline & annual & -0.54 & -0.52 & -0.53 & -0.54 & -0.53 & -0.54 & -0.10 & -0.31 & -0.41 & -0.51 \\
\hline \multirow{4}{*}{$\begin{array}{c}\text { Maximum } \\
\text { Temperature (Tmax) }\end{array}$} & pre- & -0.16 & -0.33 & -0.29 & -0.12 & -0.23 & -0.37 & 0.22 & 0.29 & 0.35 & 0.25 \\
\hline & monsoon & 0.38 & 0.44 & 0.40 & 0.51 & 0.30 & 0.25 & 0.65 & 0.58 & 0.83 & 0.61 \\
\hline & post- & -0.19 & -0.10 & 0.02 & -0.07 & 0.05 & -0.06 & 0.29 & 0.17 & 0.28 & 0.02 \\
\hline & annual & -0.06 & -0.12 & -0.01 & 0.08 & 0.05 & -0.09 & 0.45 & 0.42 & 0.54 & 0.29 \\
\hline \multirow{4}{*}{ Glacier Melt (Tmax) } & pre & -0.15 & -0.34 & 0.48 & 0.05 & -0.16 & -0.26 & 0.22 & 0.21 & 0.27 & 0.03 \\
\hline & monsoon & 0.35 & 0.39 & 0.37 & 0.49 & 0.29 & 0.23 & 0.65 & 0.61 & 0.87 & 0.65 \\
\hline & post & 0.23 & 0.45 & 0.41 & 0.37 & 0.56 & 0.52 & 0.30 & 0.37 & 0.23 & 0.12 \\
\hline & annual & 0.32 & 0.24 & 0.36 & 0.48 & 0.36 & 0.27 & 0.51 & 0.60 & 0.68 & 0.41 \\
\hline \multirow{4}{*}{ Glacier Melt (Tmean) } & pre & -0.45 & -0.42 & -0.45 & -0.31 & -0.54 & -0.38 & -0.35 & -0.47 & -0.51 & -0.51 \\
\hline & monsoon & -0.05 & -0.01 & -0.03 & 0.15 & -0.14 & -0.07 & 0.44 & 0.06 & 0.24 & -0.02 \\
\hline & post & -0.03 & -0.08 & 0.03 & -0.25 & 0.11 & -0.01 & -0.39 & -0.22 & -0.46 & -0.50 \\
\hline & annual & -0.07 & -0.03 & -0.05 & 0.11 & -0.16 & -0.09 & 0.39 & 0.01 & 0.17 & -0.09 \\
\hline \multirow{4}{*}{ Precipitation } & pre- & 0.46 & 0.73 & 0.69 & 0.83 & 0.41 & 0.59 & 0.75 & -0.22 & 0.37 & 0.21 \\
\hline & monsoon & 0.50 & 0.70 & 0.72 & 0.76 & 0.55 & 0.65 & 0.44 & -0.27 & 0.17 & 0.12 \\
\hline & post- & 0.39 & 0.52 & 0.67 & 0.62 & 0.30 & 0.45 & 0.60 & -0.09 & 0.36 & 0.16 \\
\hline & annual & 0.50 & 0.64 & 0.69 & 0.74 & 0.55 & 0.61 & 0.41 & -0.38 & 0.10 & 0.03 \\
\hline \multirow{7}{*}{ Potential Evaporation } & pre- & -0.54 & -0.54 & -0.52 & -0.37 & -0.30 & -0.40 & -0.22 & -0.22 & -0.37 & -0.54 \\
\hline & monsoon & -0.32 & -0.45 & -0.52 & -0.36 & -0.52 & -0.52 & 0.04 & -0.08 & -0.04 & -0.17 \\
\hline & post- & 0.17 & 0.47 & 0.31 & 0.25 & 0.52 & 0.53 & 0.52 & -0.02 & 0.10 & 0.27 \\
\hline & annual & -0.47 & -0.41 & -0.52 & -0.36 & -0.44 & -0.44 & 0.27 & -0.20 & -0.14 & -0.24 \\
\hline & & $p<0.001$ & & & & & & & & & \\
\hline & & $p<0.01$ & & & & & & & & & \\
\hline & & $p<0.05$ & & & & & & & & & \\
\hline
\end{tabular}

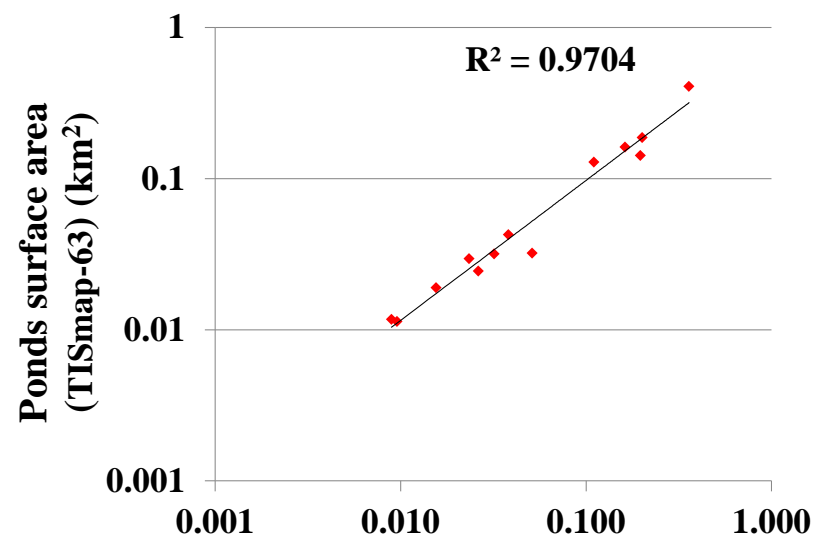

Ponds surface area

(Corona KH-4) (km²)

Figure SI1. Scatter-plot between ponds surface areas digitalized on Corona (KH-4) and TISmap-63 


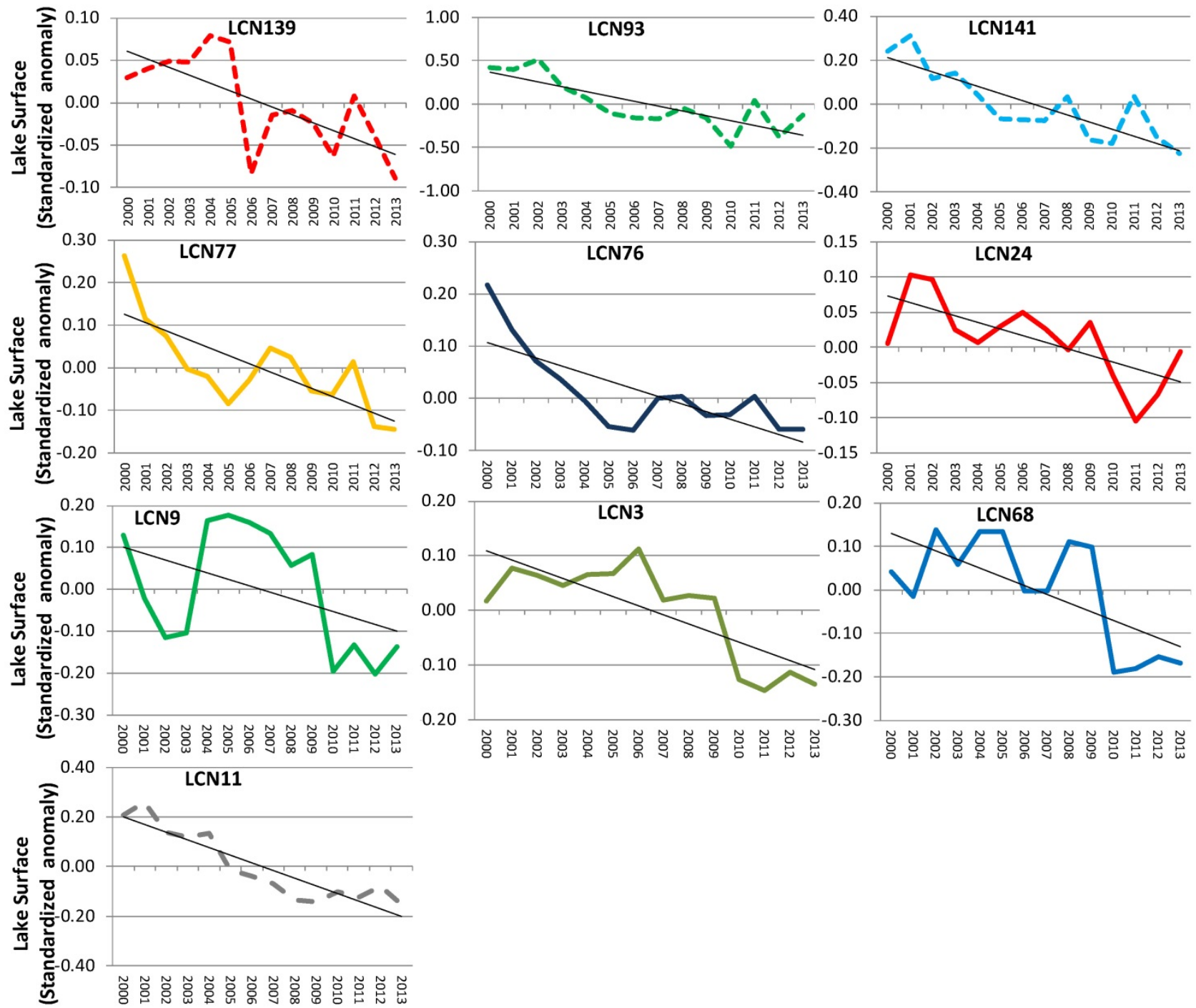

Figure SI2. Surfaces area variations during the period 2000-2013 of 10 selected ponds. Standardized anomalies (dimensionless) are computed dividing the anomalies for the standard deviation. 

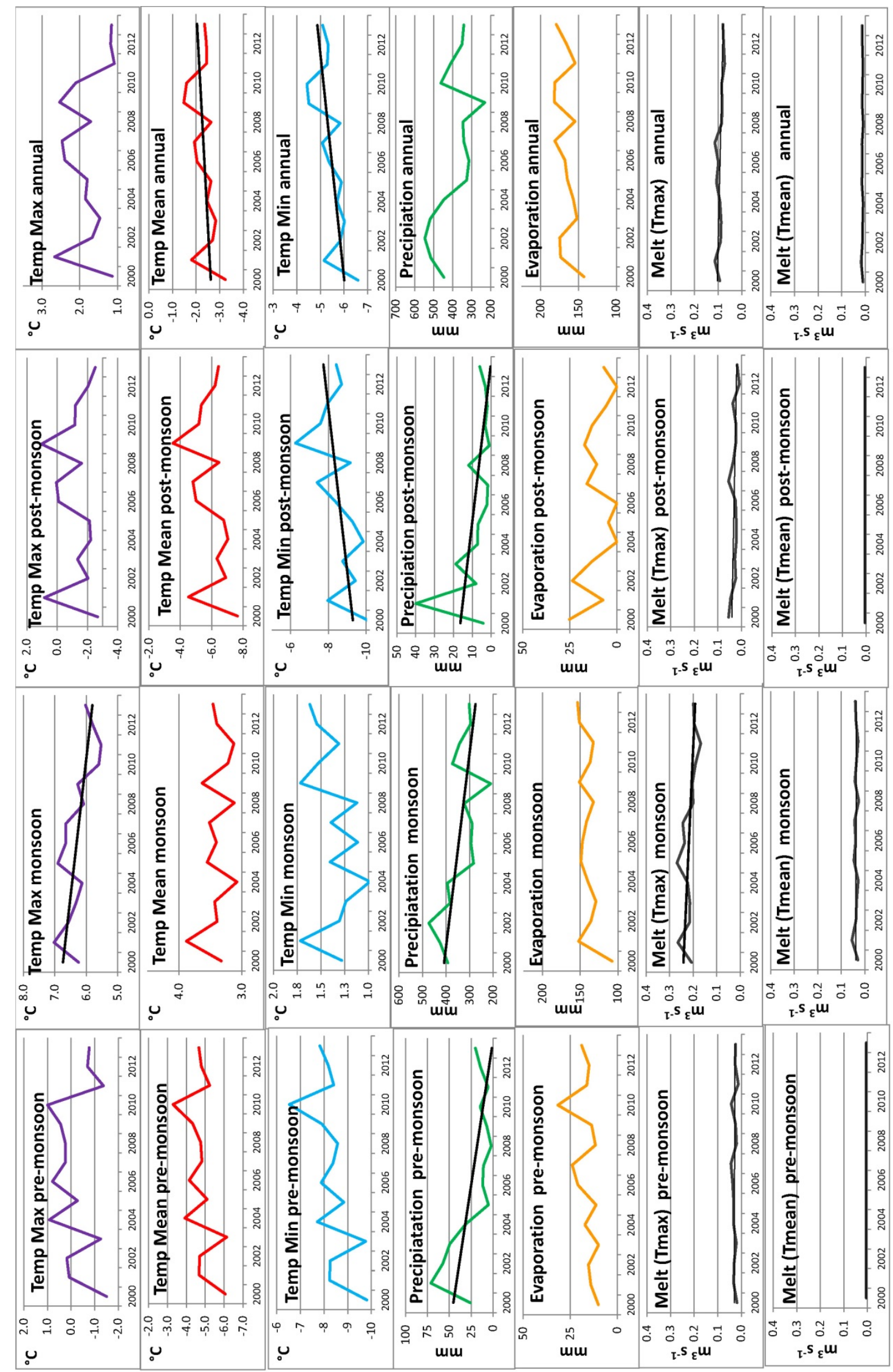

Figure SI3. Climatic trends for the period 2000-2013 at the Pyramid Laboratory (5050 m a.s.l.) for mean (Tmean), maximum (Tmax), minimum temperature, precipitation, potential evaporation, and glacier melt calculated for each glacier considering Tmax and Tmean. The regression line is indicated only in those graphs for which the trend is significant. 

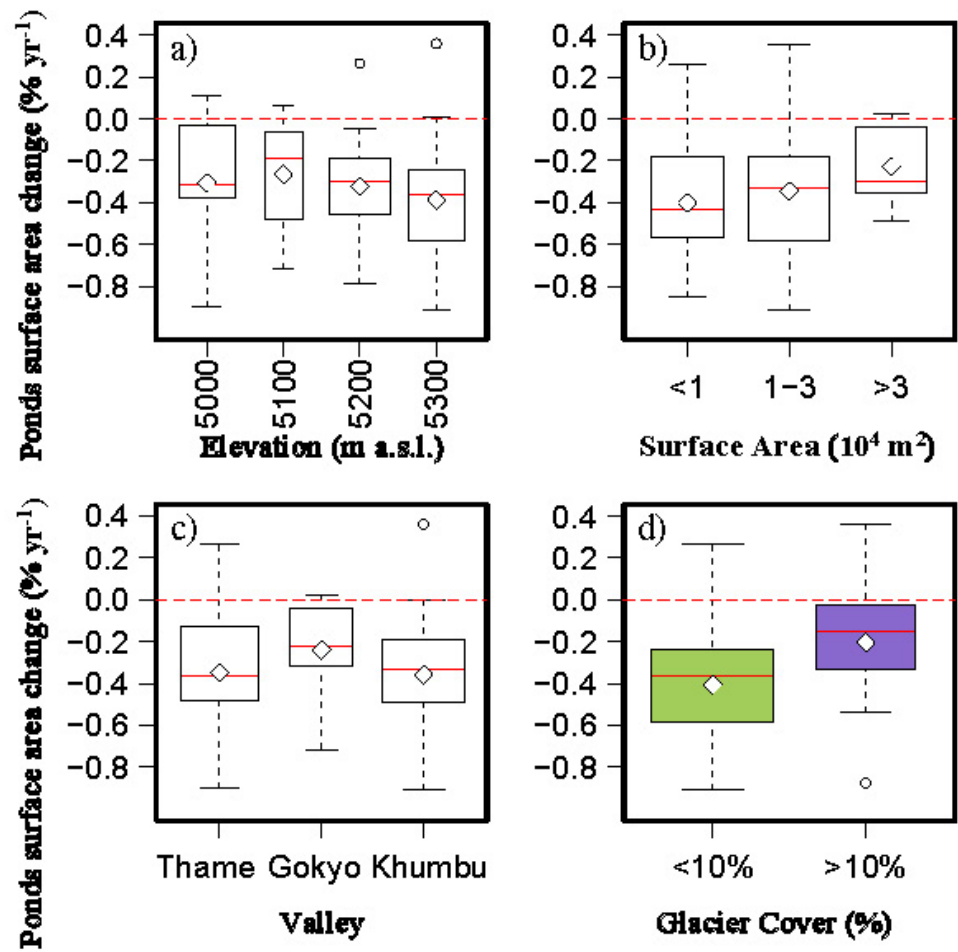

Surface Area $\left(10^{4} \mathrm{~m}^{2}\right)$

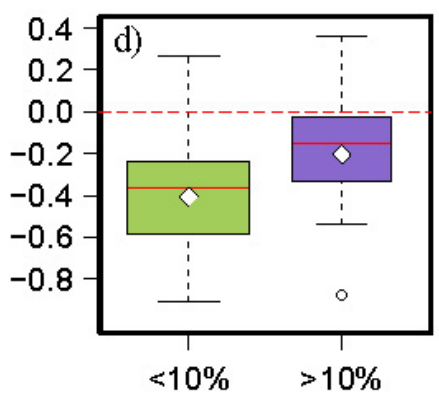

Glacier Cover (\%)

Figure SI4. Surface area changes observed during the 1992-2013 period for all 64 considered ponds in relation to certain morphological boundary conditions: a) elevation; b) pond surface area; c) valley; d) glacier cover. The white points in the boxplots indicate the mean, whereas the red lines are the median.

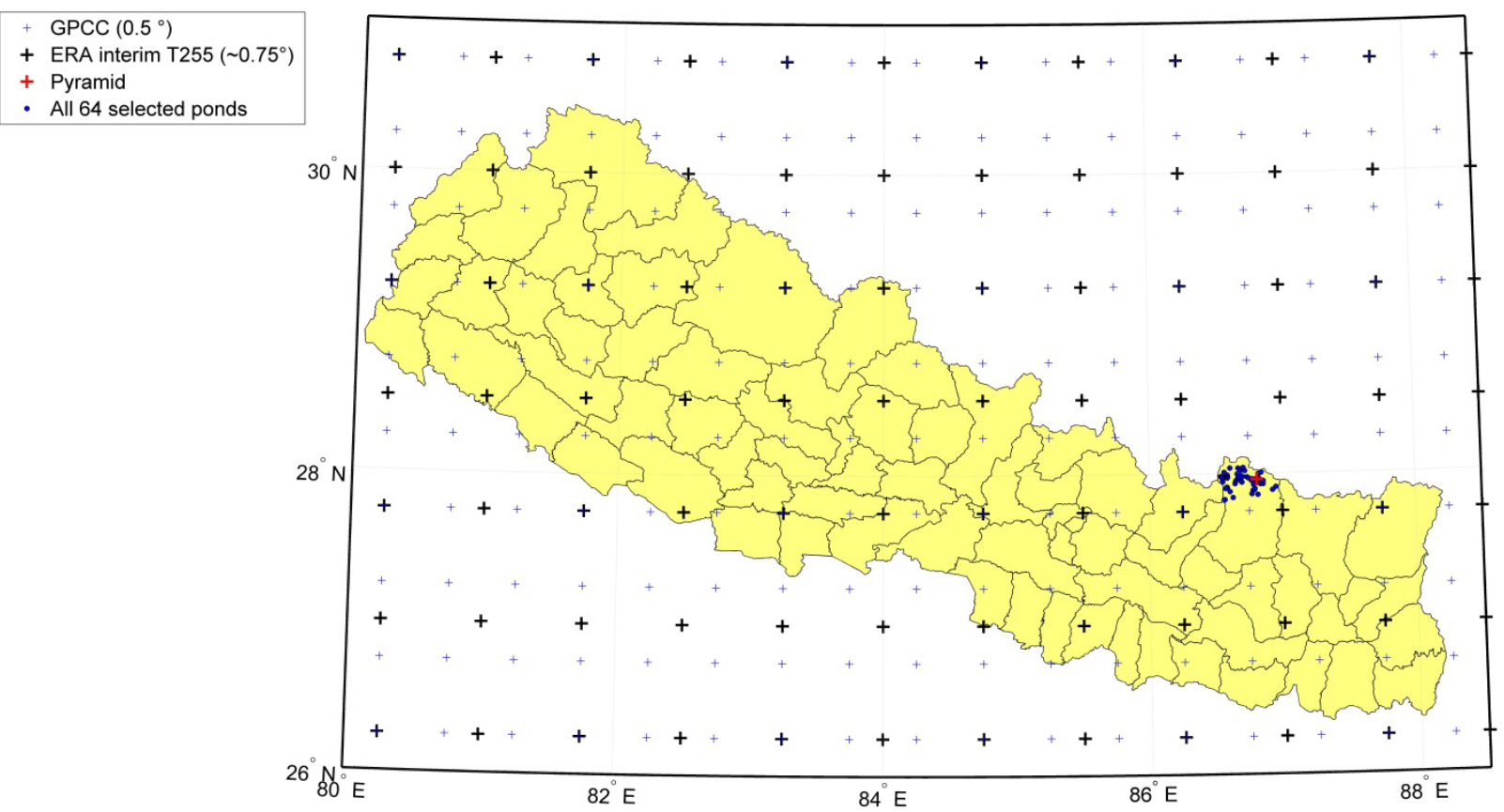

Figure SI5. Map of Nepal showing the location of 64 considered lakes in Sagarmatha National Park, Era Interim, and GPPC nodes 

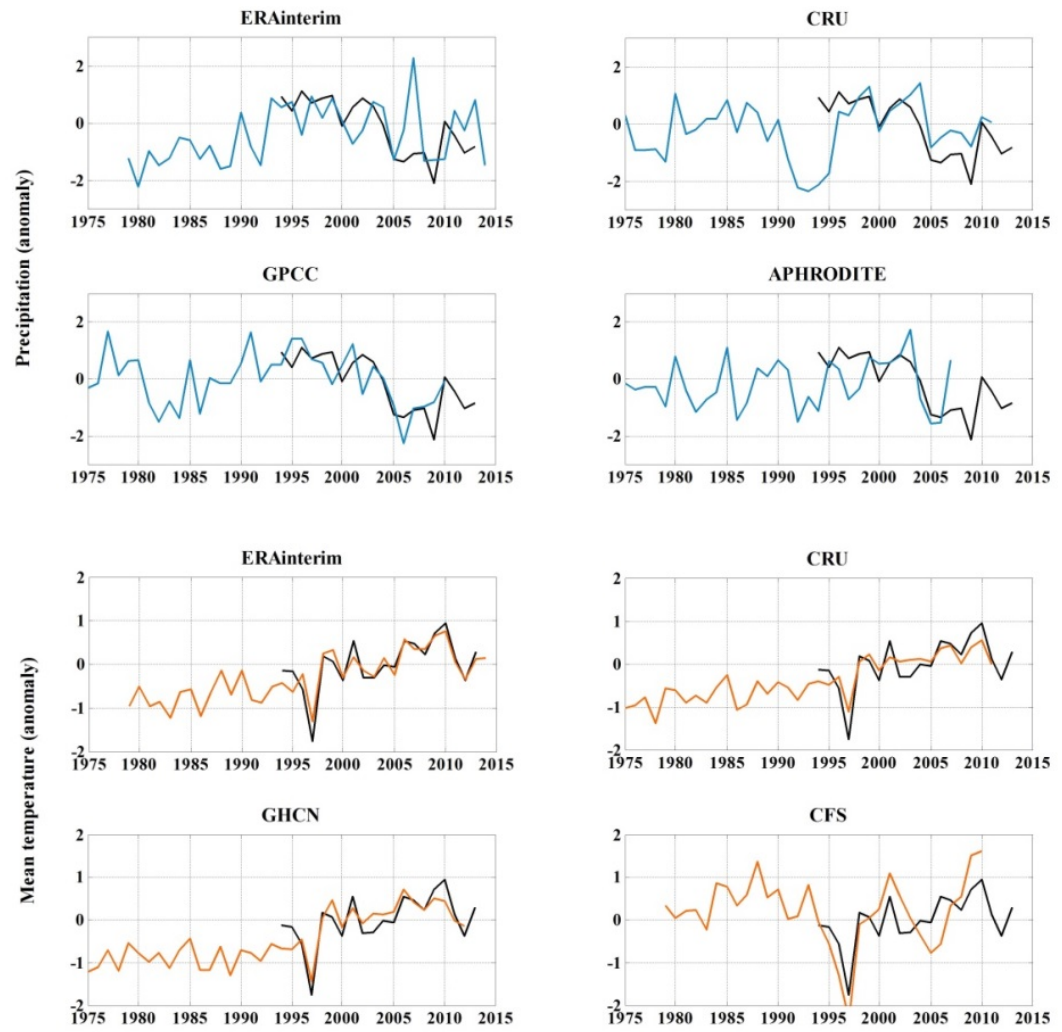

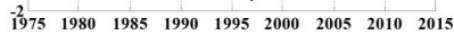

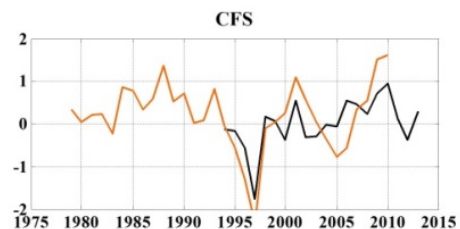

Figure SI6. Comparison between annual precipitation and mean temperature time series recorded at Pyramid station since 1994 ( black lines) with the selected regional gridded and reanalysis datasets. In Table 1 the relevant coefficients of correlation are reported
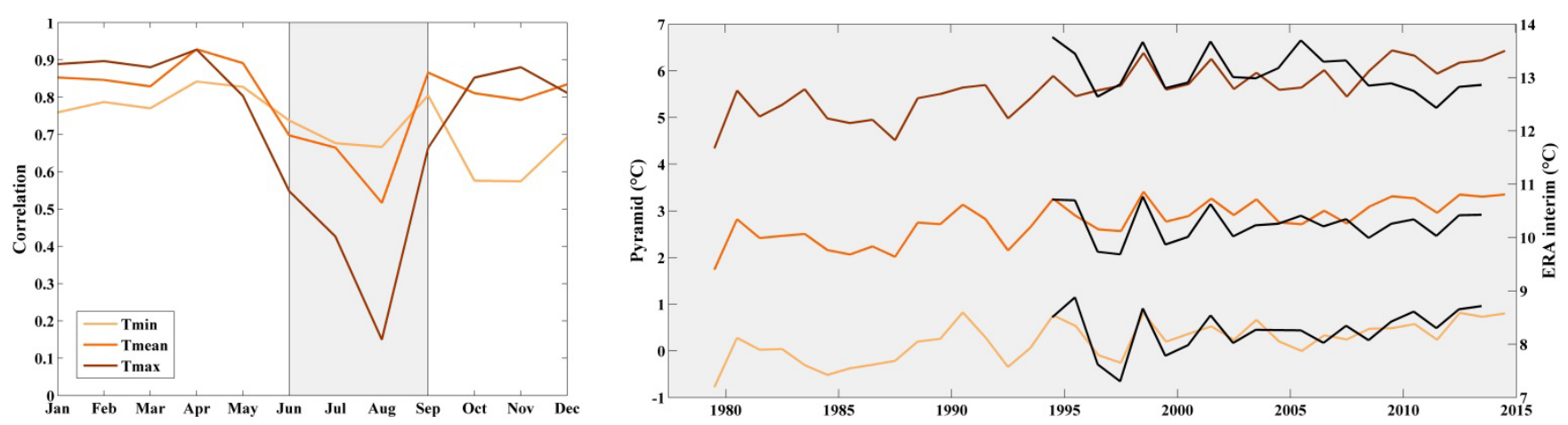

Figure SI7. a) Monthly coefficients of correlation between temperature recorded at Pyramid Station and Era Interim data (1994-2013 period)during the monsoon season. b) Temporal comparison among Era Interim and Pyramid data (black lines).

\section{References}

Dee, D. P., et al.: The ERA-Interim reanalysis: configuration and performance of the data assimilation system, Q. J. R. Meteorol. Soc., 137, 553-597, doi:10.1002/qj.828, 2011.

Fan, Y., and van den Dool, H.: A global monthly land surface air temperature analysis for 1948-present, J. Geophys. Res., 113, D01103, doi: 10.1029/2007JD008470, 2008. 
Harris, I., Jones, P. D., Osborn, T. J., and Lister, D. H.: Updated high resolution grids of monthly climatic observations - the CRU TS3.10 dataset, Int. J . Climatol., 34, 623-642, doi: 10.1002/joc.3711, 2015.

Saha, S., et al.: The NCEP Climate Forecast System Reanalysis, Bull. Amer. Meteorol. Soc., 91, 1015-1057, doi: http://dx.doi.org/10.1175/2010BAMS3001.1, 2010.

Schneider, U., Becker, A., Finger, P., Meyer-Christoffer, A., Ziese, M., and Rudolf, B.: GPCC's new land surface precipitation climatology based on quality-controlled in situ data and its role in quantifying the global water cycle. Theor. Appl. Climatol., 115,15-40, doi: 10.1007/s00704 013-0860-x, 2013.

Yatagai, A., Yasutomi, N., Hamada, A., Kitoh, A., Kamiguchi, K., and Arakawa O.: APHRODITE: Constructing a long-term daily gridded precipitation dataset for Asia based on a dense network of rain gauges, Bull. Am. Meteorol. Soc., 93, doi: http://dx.doi.org/10.1175/BAMS-D-11-00122.1, 1401-1415, 2012. 